\title{
Screen-and-treat program by point-of-care of crosuak Atopobium vaginae and Gardnerella vaginalis in preventing preterm birth (AuTop trial): study protocol for a randomized controlled trial
}

Florence Bretelle ${ }^{1,2}$, Florence Fenollar ${ }^{2}$, Karine Baumstarck ${ }^{3,4}$, Cécile Fortanier ${ }^{5}$, Jean François Cocallemen ${ }^{1}$, Valérie Serazin ${ }^{6,7}$, Didier Raoult ${ }^{2}$, Pascal Auquier ${ }^{3,4}$ and Sandrine Loubière ${ }^{3,4^{*}}$

\begin{abstract}
Background: International recommendations in favor of screening for vaginal infection in pregnancy are based on heterogeneous criteria. In most developed countries, the diagnosis of bacterial vaginosis is only recommended for women with high-risk of preterm birth. The Nugent score is currently used, but molecular quantification tools have recently been reported with a high sensitivity and specificity. Their value for reducing preterm birth rates and related complications remains unexplored. This trial was designed to assess the cost-effectiveness of a systematic screen-and-treat program based on a point-of-care technique for rapid molecular diagnosis, immediately followed by an appropriate antibiotic treatment, to detect the presence of abnormal vaginal flora (specifically, Atopobium vaginae and Gardnerella vaginalis) before 20 weeks of gestation in pregnant women in France. We hypothesized that this program would translate into significant reductions in both the rate of preterm births and the medical costs associated with preterm birth.

Methods/Design: A multicenter, open-label randomized controlled trial (RCT) will be conducted in which 20 French obstetrics and gynecology centers will recruit eligible pregnant women at less than 20 weeks gestation with singleton pregnancy and with a low-risk factor for preterm birth. Interventions will include a) an experimental group that will receive a systematic rapid screen-and-treat program from a point-of-care analysis using a molecular quantification method and b) a control group that will receive usual care management. Randomization will be in a 1:1 allocation ratio. The primary endpoint that will be assessed over a period of 12 months will be the incremental cost-effectiveness ratio (ICER) expressed as cost per avoided preterm birth before 37 weeks. Secondary endpoints will include ICER per avoided preterm birth before 24, 28 and 32 weeks, obstetrical outcomes, neonatal outcomes, rates of treatment failure and recurrence episodes for positive women. Uncertainty surrounding these estimates will be addressed using nonparametric bootstrapping and represented using cost-effectiveness acceptability curves. A total of 6,800 pregnant women will be included.

(Continued on next page)
\end{abstract}

\footnotetext{
* Correspondence: sandrine.loubiere@univ-amu.fr

${ }^{3}$ EA3279 Self-perceived Health Assessment Research Unit and Department of

Public Health, AP-HM, Aix-Marseille University, Marseille, France

${ }^{4}$ Department of Research and Innovation, Support Unit for clinical research

and economic evaluation, Assistance Publique - Hôpitaux de Marseille,

Marseille 13385, France

Full list of author information is available at the end of the article
}

\section{Biomed Central}

(c) 2015 Bretelle et al. Open Access This article is distributed under the terms of the Creative Commons Attribution 4.0 International License (http://creativecommons.org/licenses/by/4.0/), which permits unrestricted use, distribution, and reproduction in any medium, provided you give appropriate credit to the original author(s) and the source, provide a link to the Creative Commons license, and indicate if changes were made. The Creative Commons Public Domain Dedication waiver (http://creativecommons.org/publicdomain/zero/1.0/) applies to the data made available in this article, unless otherwise stated. 
(Continued from previous page)

Discussion: This appropriate randomized controlled design will provide insight into the cost-effectiveness and therefore the potential cost savings of a rapid screen-and-treat strategy for molecular abnormal vaginal flora in pregnant women. National and international recommendations could be updated based on the findings of this study.

Trial registration: ClinicalTrials.gov: NCT02288832 (registration date: 30 October 2014); Eudract: 2014-001559-22.

Keywords: Pregnancy, bacterial vaginosis, preterm birth, screen-and-treat strategy, cost-effectiveness analysis, point-ofcare, molecular diagnosis, antibiotic treatment, randomized controlled trial, Atopobium vaginae, Gardnerella vaginalis

\section{Background}

Prematurity is an important cause of death and disabilities of infants and children [1]. The preterm birth rate was approximately $7.4 \%$ in France in 2010 [2], as observed in other developed countries [3]. The presence of infection and/or inflammation during pregnancy is notably known as a major risk factor for spontaneous preterm birth [4-6]. Pregnant women with bacterial vaginosis (BV) have an increased risk of preterm birth compared to women without $\mathrm{BV}$, and the risk of preterm birth is higher if BV occurs in the early stage of pregnancy [5]. Several bacteria are associated with the diagnosis of BV [7]. However, BV is always associated with the presence of high loads of Atopobium vaginae and/or Gardnerella vaginalis $[8,9]$. Our team previously showed that high vaginal concentrations of A. vaginae and/or G. vaginalis were associated with a significantly decreased interval between preterm labor and delivery in high-risk pregnancies $[10,11]$. Therefore, the control of vaginal flora anomalies (especially A. vaginae and/or G. vaginalis) in lowrisk populations should be considered as an important aim, to see whether their treatments might prevent preterm birth.

In France, the screening (and treatment) of BV is only recommended for women with high-risk of preterm birth $[12,13]$. Because BV is asymptomatic in approximately $50 \%$ of cases [14] and early vaginal infection during pregnancy induces higher risk of obstetrical complications, one of the measures to prevent preterm birth should concern a systematic screen-and-treat strategy at the first stage of pregnancy. The standard diagnostic test is the Nugent score [13], which is laborious and not easily reproducible [9]. Such abnormalities have led some authors to propose new diagnostic tools based on molecular biological techniques $[7,9,15,16]$. The molecular approach based on the quantitative real-time polymerase chain reaction (qPCR) assay appears to be more reproducible and reclassifies a large number of flora rated as intermediate or normal on the Nugent score as true BV $[6,17]$.

Answering cost-effectiveness questions is a critical step in the translation of technological innovation findings into decision making at the public policy level. Although there is a considerable amount of literature on the economic consequences of preterm birth, little is known about the cost-effectiveness of screen-and-treat strategies for vaginal infection. To date, only one cost-effectiveness analysis of a simple screen-and-treat program for common asymptomatic vaginal infections in pregnancy has been published [18]: it showed cost savings through the first 6 years of life. In this study, the women were tested with a vaginal Gram stain and scoring criteria by the Nugent technique, which does not identify the morphotypes associated with $\mathrm{BV}$ such as A. vaginae, and the screening was performed in the second trimester of pregnancy, which is probably too late to prevent pregnancy complications.

All these considerations may lead to questions on the cost effectiveness of a systematic screen-and-treat program during the first trimester for flora vaginal anomalies among pregnant women with a low-risk of preterm birth, taking into account advances in diagnostic tools, bacteria species targeted and antibacterial treatments. For this purpose, a multicenter, randomized controlled study was designated to assess the cost effectiveness of the innovative screening for $A$. vaginae and G. vaginalis vaginal portage using a molecular quantification method by point-of-care with an appropriate treatment for positive cases, compared to a usual care strategy in pregnant women at less than 20 weeks of gestation. Several obstetrical and neonatal secondary outcomes are also considered, as well as rates of treatment failure and recurrence episodes.

\section{Methods/Design \\ Design}

A multicenter, open-label randomized controlled, twoparallel group study was designed in which pregnant women who attend prenatal care consultations before 20 weeks' gestation at French obstetrics and gynecology centers are randomized between two management strategies: a systematic vaginosis screen-and-treat strategy (experimental group) and usual care management (control group). The study protocol was designed using the recommendations of the Consolidated Standards of Reporting Trials (CONSORT) statement and according to the guidelines of cost-effectiveness studies of the French Health Authority [Haute Autorité en Santé, Choix méthodologiques pour l'évaluation économique à 
la HAS http://www.has-sante.fr/portail/jcms/c_1120711/ choix-methodologiques-pour-l-evaluation-economique-ala-has]. Timing and phasing after eligibility checks are shown in Fig. 1.

\section{Partners}

This work is supported by an institutional grant from the French 2014 National Program of Cost-effectiveness Research (Programme de Recherche Médico-Economique, FINESS number 130789049). The recruiting will take place in 20 French obstetrics and gynecology centers. The molecular analyses will be performed in two point-of-care laboratories. The methodological support will be provided by the Clinical and Cost-Effectiveness Research Unit (Assistance Publique - Hôpitaux de Marseille, AP-HM, France), and the Clinical Investigation Unit (Centre d'Investigation Clinique, AP-HM, France). The central pharmacy of AP-HM is in charge of the assignment, allocation and delivery of the devices. All the details are provided in Table 1.

\section{Inclusion and exclusion criteria}

The details of the inclusion and exclusion criteria are provided in Table 2. The main inclusion criteria are that women must have less than 20 weeks of gestation, with singleton pregnancy, they must be symptomatic or nonsymptomatic with regard to their diagnosis of $\mathrm{BV}$, and they must not have high-risk factors of preterm birth. The main exclusion criteria are having known conditions at the time of recruitment that have either increased risk of spontaneous preterm birth (previous preterm birth, uterine malformation, or multiple pregnancy) or that may need preterm delivery due to medical indication: hypertension, diabetes, fetal malformation, increased risk for preeclampsia (or other conditions that the investigators may consider).

\section{Interventions}

Experimental group: screen-and-treat strategy

Pregnant women assigned to the intervention group are asked for a self-collected vaginal swab at randomization

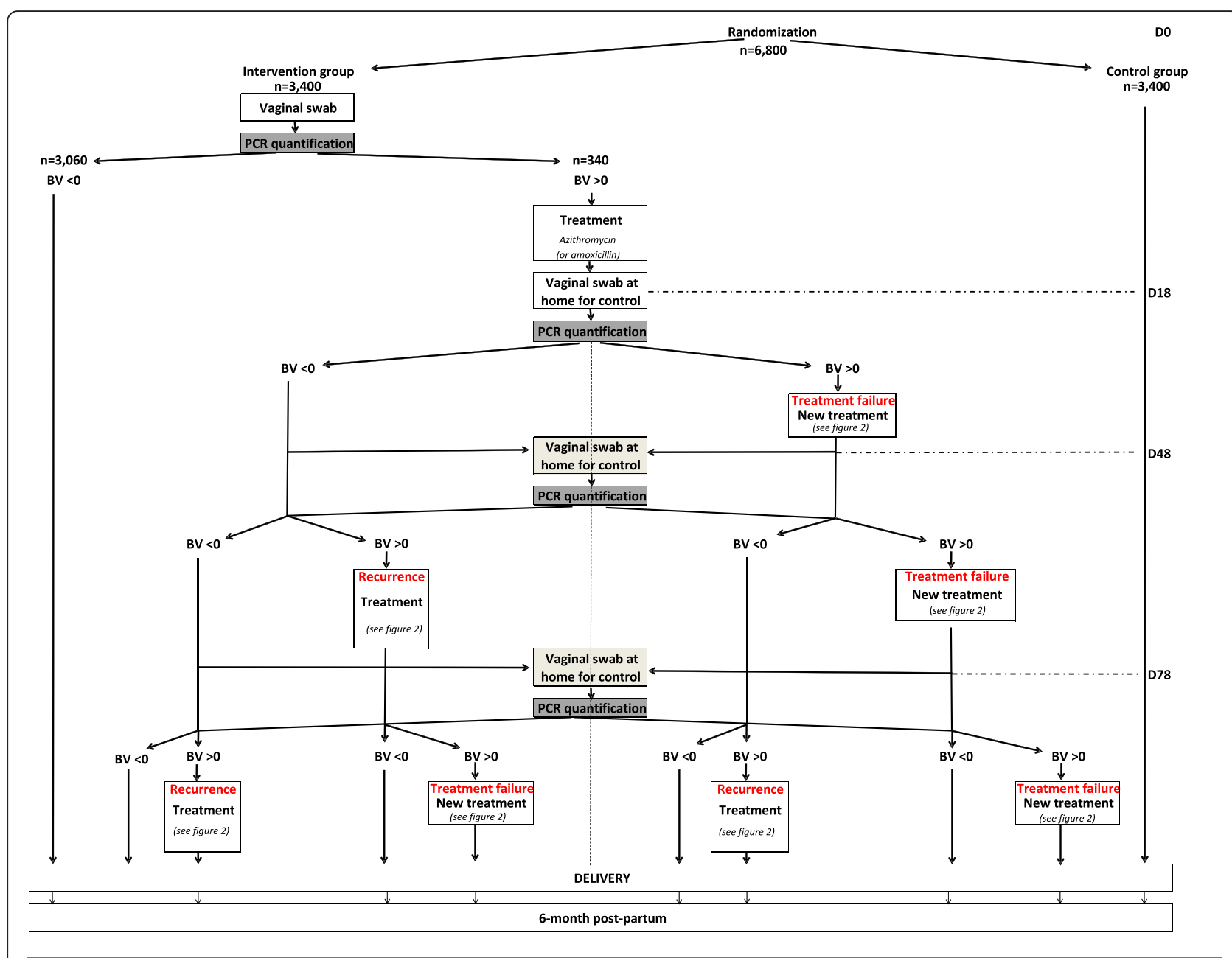

Fig. 1 Schema of timing and phasing - AuTop Study 
Table 1 French partners

\begin{tabular}{|c|c|}
\hline Gynecologists & Center/department \\
\hline Pr Florence Bretelle & Coordinating investigator. Public academic teaching hospital Nord, Marseille \\
\hline Dr Hélène Heckenroth & Public academic teaching hospital La Conception, Marseille \\
\hline Dr Raoul Desbriere & Private Hospital Saint Joseph, Marseille \\
\hline Dr Nadia Slim & Private Hospital Bouchard, Marseille \\
\hline Dr Nawal Chenni-Asselah & Public hospital, Aubagne \\
\hline Dr Xavier Danoy & Public academic hospital, Aix-en-Provence \\
\hline Dr Franck Mauviel & Public academic hospital, Toulon Sainte Musse \\
\hline Pr André Bongain & Public academic teaching hospital, Nice \\
\hline Pr Pierre Mares & Public academic teaching hospital, Caremeau \\
\hline Pr Patrick Rozenberg & Public academic hospital, Poissy-Saint-Germain \\
\hline Dr Thomas Schmitz & Public academic teaching hospital Robert Debré, Paris \\
\hline Pr Alexandra Benachi & Public academic teaching hospital Antoine Béclère, Clamart \\
\hline Pr Marie-Victoire Senat & Public academic teaching hospital Kremlin-Bicêtre, Kremlin-Bicêtre \\
\hline Pr Bassam Haddad & Public academic hospital, Créteil \\
\hline Dr Jean-Pierre Ménard & Protection Maternelle Infantile, Val de Marne \\
\hline Pr Gilles Kayem & Public academic teaching hospital Armand Trousseau, Paris \\
\hline Pr Loic Sentilhes & Public academic teaching hospital, Angers \\
\hline Pr Céline Chauleur & Public academic teaching hospital, Saint-Etienne \\
\hline Dr Jean-Luc Volumenie & Public academic teaching hospital, Martinique \\
\hline Dr Philippe Kadhel & Public academic teaching hospital, Pointe-à-Pitre \\
\hline Clinical microbiologists & Center/department \\
\hline Pr Florence Fenollar & Fédération de Microbiologie Clinique. Public academic teaching hospital la Timone, Marseille \\
\hline Dr Valérie Serazin & Service de Biologie Médicale - UF de Biologie moléculaire. Public general hospital, CHI Poissy St Germain \\
\hline Methodology team & Center/department \\
\hline Pr Pascal Auquier & Public health, public academic teaching hospital, Marseille \\
\hline Sandrine Loubière \& Cécile Fortanier & Health economy, public academic teaching hospital, Marseille \\
\hline Dr Karine Baumstarck & Clinical research unit, public academic teaching hospital, Marseille \\
\hline Dr Nathalie Lesavre & Clinical investigation center, public academic teaching hospital, Marseille \\
\hline Dr Stéphane Honoré \& Dr Anita Cohen & Pôle Pharmacie, public academic teaching hospital, Marseille \\
\hline
\end{tabular}

(Time 0). The self-collected vaginal swab has previously been demonstrated to have high validity and reliability compared to a practitioner-collected swab [17]. The swab will be immediately tested for $A$. vaginae and $G$. vaginalis using a systematic point-of-care screening test. Quantitative molecular analyses will be performed in laboratories that have experienced and national accreditation to realize point-of-care techniques. The quantitative real-time PCR (qPCR) method used to diagnose BV was previously described [11]. The result is reported as copies of microorganism DNA per $1 \mathrm{~mL}$ of vaginal suspension [9]. According to previous works $[9,11]$, BV will be defined by an $A$. vaginae load $\geq 10^{8}$ copies/mL and/or a $G$. vaginalis load $\geq 10^{9}$ copies $/ \mathrm{mL}$. The conclusion will be feedback of the positive or negative test result to the practitioner within less than 24 hours. In cases of positive diagnosis, the pregnant woman will be referred to an obstetrician, and an appropriate antibiotic treatment will be provided. The first intention for treatment of A. vaginae and G. vaginalis will be azithromycin (single dose, $1 \mathrm{~g}$ at day 1 and $1 \mathrm{~g}$ at day 3 ). The second choice for antibiotic treatment will be amoxicillin $2 \mathrm{~g}$ per day during 7 days in cases of known intolerance of azithromicin. The following procedure for women who are diagnosed positive is to perform a series of three successive screening controls until 28 weeks to detect either the failure of antibiotic treatment or a recurrence (that is, reappearance of bacteria from a control vaginal swab after therapy is stopped) (see Fig. 1). In cases of recurrence or treatment failure, a new antibiotic treatment will be provided, based on protocol guidelines (see Fig. 2). For women with a first negative diagnosis, the usual care management will be proposed. 
Table 2 Selection criteria

Inclusion criteria
Woman $\geq 18$ years of age
Woman less than 20 weeks of gestation
Woman with singleton pregnancy
Woman without history of preterm birth or late miscarriage
Woman with low-risk factor of preterm birth (absence of diabetes,
systemic lupus erythematosus, treated hypertension, fetal malformation,
cervical conization, or multiple pregnancy)
Woman affiliated to or beneficiary of a social security system
Woman who have signed written informed consent
Exclusion criteria
Woman more than 20 weeks of gestation
Minor woman or woman deprived of their freedom by a court/
administrative decision or woman under legal protection
Woman who present high-risk factor of preterm birth or late
miscarriage
Woman with extrauterine pregnancy
Woman with non-evolutive pregnancy
Woman who have received antibiotic treatment in the week before
inclusion
Woman misunderstanding the written and spoken French language
Subject participating in another biomedical research protocol

\section{Control group: usual care management}

No systematic vaginosis screening will be performed on the women assigned to the control group according to national and international guidelines [13, 19]. Usual pregnancy care includes 6 to 8 obstetrical consultations, no systematic vaginal swab, three ultrasound scans, 1st trimester Down syndrome screening and blood sampling for toxoplasmosis, syphilis, rubella, and complete blood group testing.

\section{Recruitment}

Eligible women will be invited to take part in the study during a routine prenatal consultation planned in the first trimester of their pregnancy. The women who meet all the inclusion criteria will be randomized into one of the two groups after completing the informed consent form.

\section{Randomization}

Computer-generated randomized lists were drawn up before the beginning of the study, using a permuted block design, under the responsibility of the clinical research unit (AP-HM). The randomization was stratified by center (1:1 allocation ratio).

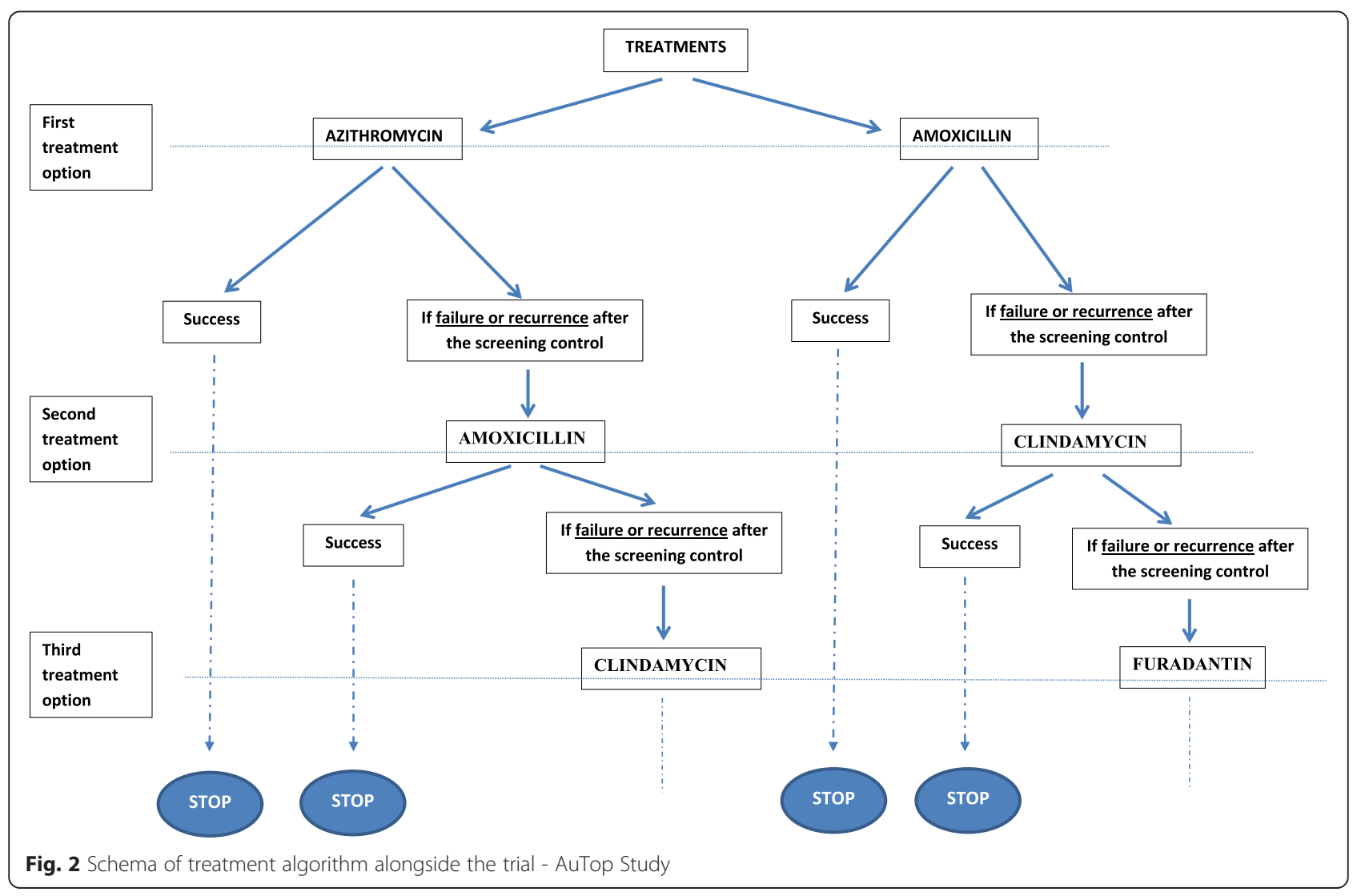




\section{Endpoints}

\section{Primary endpoint}

The primary endpoint is the incremental cost-effectiveness ratio (ICER), expressed as the extra cost per additional preterm birth avoided before 37 weeks. The effectiveness criterion has been discussed and consensually approved by all the study's main partners (gynecologist coordinator and co-coordinators, biologists, health economist and methodologist). We can assume that this intermediary criterion properly reflects short- and long-term prognosis of the children [20,21].

\section{Secondary endpoints}

The secondary endpoints are as follows:

1. ICER per preterm birth avoided before 26, 28, 32 and 34 weeks.

2. Obstetrical outcomes: rates of preterm birth before $26,28,32,34$, and 37 weeks of gestation, spontaneous abortion (between 14-22 weeks), late miscarriage (between 22-24 weeks), premature rupture of membranes, severe intrauterine growth restriction, preterm labor, duration of the woman's hospitalization (that is hospitalization for delivery or preterm labor and potential previous hospitalizations due to gynecologic complications during pregnancy).

3. Neonatal outcomes: neonatal mortality, neonatal morbidity (respiratory distress syndrome, bronchopulmonary dysplasia, necrotizing enterocolitis, periventricular leukomalacia), transfer to a neonatal intensive care unit (duration), mechanical ventilation (duration), congenital anomalies and duration of the newborn's hospitalization.

4. Treatment effectiveness: rate of recurrence (defined as a positive control vaginal swab using qPCR after the negativation of a precedent control vaginal swab), rate of treatment failure (defined as $A$. vaginae $>10^{8}$ copies $/ \mathrm{mL}$ and/or a G. vaginalis load $\geq 10^{9}$ copies/mL from a control vaginal swab) and side effects associated with treatment.

5. Other health care utilization: all the mother's use of health care during the whole study period (for example, gynecologists and general practitioner consultations, hospital admission, clinical examinations and medications), as well as health care for the newborn (including neonatal care, re-hospitalization, medications, planned and non-planned consultations with pediatric practitioner or other specialists).

\section{Data collection and follow-up}

All data will be recorded from an electronic case report form (eCRF) specifically elaborated for the study (eCRF CleanWEB, Telemedicine Technologies S.A.S., www. tentelemed.com, 2015) and will be recorded at four specific study's times as follows: randomization (T0), baseline assessment (T1), delivery (T2), and at 6 months after delivery (T3). All assessments are based either on medical files (pregnancy and delivery characteristics, obstetrical and neonatal outcomes), face-to-face questionnaires (smoking and alcohol habits, personal hygiene, pregnancies history or symptoms, and concomitant treatments such as treatment with pessary or progesterone), phone calls (to collect data on vaginal symptoms or potential side-effects of antibiotic) or self-report (health outcomes of their infant and health service use during the 6 months following the initial hospitalization). Details of the data recorded at the different times of the study are given in Table 3.

\section{Pharmaceutical aspects}

Experimental drugs, azithromycin $250 \mathrm{mg}$ oral tablets $\left(\right.$ ZITHROMAX $^{\mathrm{Tm}}$ ) and amoxicillin $500 \mathrm{mg}$ oral capsules

Table 3 Data collection, instruments and assessment times

At randomization (TO)

At baseline assessment (during screening and treatment phases - T1)

At delivery (T2)

At 6 months after delivery (T3)
Data on the health status of the participant and pregnancy characteristics will be collected from medical files of the practitioners. A face-to-face questionnaire will be also completed by all women and filled out by the midwife/obstetrician to collect specific data on demographics characteristics, smoking and alcohol habits, previous pregnancies and personal hygiene. The first vaginal swab will be collected by the practitioner and send to one of the two point-of-care laboratories associated to the study.

Subsequent vaginal swabs will be realized during either routine pregnancy consultation or at the woman's home depending on each participant schedule. In this later case, the sample will be sent by the women to the referent POC laboratory using a stamped, self-addressed envelope. Symptoms and potential side effects of antibiotics will be collected via a telephone interview. Participants will be informed via a phone call for subsequent vaginal swabs and treatment intake if needed.

All relevant clinical and obstetrical outcomes during pregnancy will be collected from the medical files. To complete data collection, a face-to-face interview with women around delivery phase will be scheduled. All relevant data such as pregnancy complications, hospitalizations, delivery characteristics (including birth weight, terms at delivery, or fetal death) will be collected.

Participants will be provided with a questionnaire, on which they will be asked to record all health outcomes of their infant and associated health service use. 
$\left(\right.$ CLAMOXYL $\left.^{\mathrm{mm}}\right)$, will be packaged and labeled by the "Unité d'Expertise Pharmaceutique et Recherche Biomedicale" pharmaceutical unit of the Hospital Pharmacy of AP-HM and distributed to the dispensing hospital pharmacy of each investigating center. At the end of the study, used and unused treatments will be returned to the dispensing pharmacy and destroyed.

\section{Sample size}

The sample size was calculated from the expected differential ICER per preterm birth avoided between the two groups. In accordance with Briggs [22], the following hypothesis is stated: with an expected incremental rate of preterm birth of $1.3 \%$ (4.3\% in the control group [23] and $3.0 \%$ in the experimental group), an expected incremental cost of 230 euros (including cost of initial and following point-of-care tests and cost of treatments for $10 \%$ of the women [24]), and an expected differential ICER at 22,500 euros corresponding to the avoided cost of a preterm birth before 37 weeks [25], with an $80 \%$ statistical power and a threshold for statistical significance set at a $P$ value of 0.05 , and assuming that a potential $20 \%$ of patients will be lost to follow-up, these calculations showed that 6,800 patients are needed (3,400 per group). Considering the potential of inclusion of each participating center, the inclusion duration will be planned over a 12-month period. The maximal period of participation for the included women is 12 months.

\section{Statistical analysis}

The data will be analyzed using SPSS version 20.0 software. Statistical significance is defined as $P<0.05$. The methodology will be based on the Consolidated Standards of Reporting Trials Statement (CONSORT, http:// www.consort-statement.org/consort-statement/) [26]. The full analysis population (including all subjects who will be randomized and will be at least evaluated at baseline) will be used in the primary analysis and the per protocol population (including all subjects who will be randomized and will not have major protocol deviations) will be used in the secondary analysis to assess the reliability of the results. Finally, missing data will be handled where possible using multiple imputations, and a sensitivity analysis will be performed. No interim analysis is planned.

The normality of the parameters will be estimated using frequency histograms and the Shapiro test. In case of nonparametric distribution, the data will be log transformed to obtain a normal distribution or nonparametric bootstrapping performed for cost data. In accordance with the distribution of the parameters, the baseline parameters will be presented separately for the control group and the experimental group: mean (standard deviation) or median (interquartile range) for continuous variables, proportions for categorical variables. Then, data will be compared between the two groups using Student's t-test for continuous variables (durations), and chi-square or Fisher's exact tests for categorical variables.

\section{Cost-effectiveness analysis}

Incremental cost-effectiveness ratios (ICER) will be used to compare the cost and effectiveness of the experimental strategy with usual care. The ICER is the ratio of the difference between groups in costs to the difference in effectiveness. The difference in effectiveness equals the number of preterm births averted and is calculated as the number of preterm births in the screening group minus the number of preterm births in the control group. Thus, the ICER provides information on the potential acceptability of the intervention for decision makers. The costs perspective taken in our economic analysis is that of the healthcare payer. The time horizon started from the first prenatal consultation before the 20 weeks of gestation and ended at 6-months of age or death. The healthcare costs included are those that are likely to differ across the intervention and control groups. In our study, these costs are those associated with: screening using the point-of-care procedure (quantitative molecular analysis), control vaginal swabs for positive women, antibiotic treatments, antenatal hospital admissions, physicians' consultations, management of complications, as well as neonatal costs for full term infants and preterm infants. Unit costs for health service use will be estimated using data from the French National Hospital Database (Programme de médicalisation des systèmes d'information, PMSI) and the National Tariff. Treatment costs were obtained from the French register of pharmaceutical specialties, an online database of information on healthcare products. The cost of the POC analysis using quantitative real-time PCR (qPCR) will be calculated using micro-costing technique, which is particularly well suited to evaluating the cost of an emerging technique [27]. The volume of resources used will be determined by direct observation of each stage of each testing procedure. Fixed and variable costs will be included. All resources will be valued in 2015/2016 euros, and the 12-month trial means there is no requirement to apply discounting. As the cost of preterm birth differs significantly according to the gestational age at birth [28-30], we will attempt to address this issue by calculating an ICER for specific birth gestational ages. Probabilistic sensitivity analyses, using the non-parametric bootstrap method, will be carried out to generate mean expected ICERs and to determine whether uncertainty or variation in the data used affect the ICERs [31]. In addition, cost-effectiveness acceptability curves were constructed to represent decision uncertainty surrounding cost-effectiveness estimates [32]. 


\section{Ethical aspects, laws and regulations}

The study will be conducted in accordance with the Helsinki declaration and the French laws and regulations (Code de la Santé Publique, article L.1121-1/Loi de Santé Publique n²004-806 du 9 Août 2004 relative à la politique de santé publique et ses décrets d'application du 27 Août 2006) and the International Conference on Harmonization (ICH) E6 Guideline for Good Clinical Practice. Regulatory monitoring will be performed by the sponsor. French ethics committee and French drug and device regulation agency approved conduct of the AuTop study at all sites (registered number respectively: Comité de Protection des Personnes Sud Méditerranée, reference number 14.026 and Agence Nationale de Sécurité du Médicament, reference number 140500A-41). This trial was registered into the government clinical trials under the identifier number NCT02288832 (ClinicalTrials.gov). Written Informed consent will be obtained from all subjects before inclusion.

\section{Discussion}

This is the first large randomized controlled trial assessing the cost-effectiveness of a screen-and-treat program of molecular flora vaginal anomalies during the first trimester in pregnant women with low risk of preterm birth. This study was designed specifically to inform healthcare decision making, in an international context where the diagnosis of BV in pregnant women and its subsequent management care are controversial [33-37]. Several reasons could be stressed to explain these diverging opinions.

Firstly, there was a real lack of accuracy of diagnostic tools at the time of some studies. Most of the studies cited earlier focused on the Nugent score, which has some limitations. It must be performed on a fresh swab, and any delay in transporting the swab makes the test difficult to perform, its scoring requires experienced microbiologists to ensure consistency, and some pathogens associated with BV are not identified by such a technique, in particular A. vaginae [9]. Recent studies have demonstrated that molecular biology techniques have both higher sensitivity and specificity for the diagnosis of BV $[7,9,15,16]$ compared to the Nugent score and can detect several bacterial series for BV.

The second point concerns the appropriateness of antibiotic treatment $[13,19]$. The nature of the antibiotics (such as metronidazole or clindamycin, pro- or prebiotics) and their modes of administration (oral versus vaginal) vary considerably between existing studies. Few studies have controlled for treatment efficacy [38]. In addition, although the frequency of recurrences after antibiotic treatment is high (from $28 \%$ to $50 \%$ ), depending on the nature of the treatment and the length of time from its intake [39], most of studies did not consider this risk of recurrence. In the present project, we propose to use azithromycin because of its effectiveness on $A$. vaginae and $G$ vaginalis with a lifetime that allows us to reduce the treatment duration and to increase the adherence rate [40].

The third point concerns the delay between BV and treatment. In cases of late diagnosis, and therefore delayed treatment, BV has already been established and can lead to obstetrical complications involving preterm birth [41]. In this present study, we have considered that the point-of-care test will be an interesting approach to minimize the feedback of the vaginal swab to the practitioner in order to rapidly prescribe the antibiotic treatment in cases where tests are positive. In our trial, the short window for providing a diagnostic result also appears essential for minimizing the number of women lost to follow-up who may benefit from an early antibiotic treatment. Indeed, this rate of "lost to follow-up patients" is important in this disease where asymptomatic disease, anxiety and low socioeconomic status are clearly identified risk factors [9].

Finally, the gestational age at inclusion is the greatest limitation of previous published studies. Due to the lack of data concerning the early phase of pregnancy, the authors may have underestimated the effectiveness of a universal screen-and-treat program in reducing the rate of "very preterm birth" (28 to 32 weeks). These subdivisions into "very preterm" and "moderate" or "late preterm" (that is, 32 to less than 37 completed weeks of gestation) are important since decreasing gestational age is associated with increasing mortality, intensity of neonatal care required, and hence increasing costs [42].

The main purpose of AuTop trial is to support at a nationwide level the feasibility, acceptability, and costsavings of a routine point-of-care molecular diagnosis at the first stage of pregnancy. Although it is well known that the consequences in terms of morbidity and mortality of preterm birth are important and entail a significant economic burden, several studies have demonstrated that a large share of the incremental cost per surviving preterm infant is represented by neonatal care [43, 44]. The average length of this neonatal care can vary from 3 days to 6 months for very preterm births (including potential immediate re-hospitalization, programmed consultations and bronchiolitis prevention measures). We then decided to focus on this time horizon for the costeffectiveness analysis.

In Kiss et al. [23], the prevalence of BV was around $7 \%$ in their study population (which included women without subjective complaints, that is, contractions, vaginal bleeding, or symptoms suggestive of vaginal infection, or women with multiple pregnancies), with less than $2.3 \%$ having an obstetric history at inclusion. It is well known that the ethnic origin influences the 
prevalence of bacterial vaginosis in pregnancy. In Kiss's RCT, the population was $98 \%$ white ethnic origin. In our study population, we will expected less than $65 \%$ for the white ethnic group, based on our previous RTC conducted in the same French centers [11]. Consequently, we think that a prevalence of $\mathrm{BV}$ of $10 \%$ in a population with or without clinical symptoms of BV and well balanced in terms of ethnic origins is not so optimistic. Our hypothesis of a decrease in the rate of preterm births from $4.3 \%$ to $3.0 \%$ seems to be a small expected gain in the rate of preterm births avoided. In fact, a mean difference of $1.3 \%$ is actually a very large difference for pregnant women and decision makers, and should be sufficient to prevent major healthcare expenditure. Decision analysis is particularly useful when the difference in outcomes between strategies is small, and can provide insight into the costs contributing to the public decision-making process.

Given the need for scientific evidence (in terms of both efficacy and economic) regarding bacterial vaginosis screening in a population with low-risk factors for preterm birth, our analysis should be useful for clinicians and other healthcare decision makers involved in managing care of pregnant women.

\section{Trial status}

At the time of manuscript submission, the status of the trial is yet recruiting. The inclusion of participants started in March 2015.

\section{Abbreviations \\ AAP: American Academy of Pediatrics; ACOG: American Congress of Obstetricians and Gynecologists; ANSM: Agence Nationale de Sécurité du Médicament; AP-HM: Assistance Publique-Hôpitaux de Marseille; BV: bacterial vaginosis; CDC: Centre for Disease and Controls; CONSORT: Consolidated Standards of Reporting Trials; DNA: deoxyribonucleic acid; eCRF: electronic case report form; HAS: Haute Autorité en Santé - French National Authority for Health; ICER: incremental cost effectiveness ratio; ICH: International Conference on Harmonization; PCR: polymerase chain reaction; SPSS: Statistical Package for the Social Sciences; PMSI: Programme de médicalisation des systèmes d'information; RCT: randomized controlled trial.}

\section{Competing interests}

The authors declare that they have no competing interests.

\section{Authors' contributions}

FB, CF, JFC, FF, VS, DR, KB, PA and SL conceived and/or designed the study, including the power calculation. CF and JFC acquired legal authorizations. The study protocol and manuscript have been drafted by FB, CF, SL, KB and PA. All authors read and approve the final manuscript.

\section{Acknowledgements}

This work is supported by institutional grants from the French 2013 Programme de Recherche Médico-Economique (PRME). The sponsor was represented by Assistance Publique, Hôpitaux de Marseille, France, and its role was to control the appropriateness of ethical and legal considerations. The authors are grateful to all the investigators for their participation in the study. We thank Stéphane Honoré and Kahena Amichi for their participation and collaboration during the study conception.

\section{Sponsor and funder}

Trial Sponsor: Assistance Publique-Hôpitaux de Marseille (AP-HM, France) Sponsor's Reference: FINESS 13078649

Contact name: Kahena $\mathrm{AMICH}$

Address: Direction de la Recherche Clinique et de I'Innovation, 80, rue Brochier, 13354 MARSEILLE Cedex 05, France.

Phone: +33(0) 491381966

Email: kahena.amichi@ap-hm.fr

This funding source had no role in the design of this study and will not have any role during its execution, analyses, interpretation of the data or decision to submit results.

\section{Author details}

'Department of Gynaecology and Obstetrics, Gynépole, Marseille, Pr Boubli, Hôpital Nord, Assistance Publique-Hôpitaux de Marseille (AP-HM), AixMarseille Université, Marseille, France. ${ }^{2}$ Aix-Marseille Université, Unité de Recherche sur les Maladies Infectieuses Tropicales et Emergentes, UM63, CNRS 7278, IRD 198, INSERM 1095, Marseille, France. ${ }^{3}$ EA3279 Self-perceived Health Assessment Research Unit and Department of Public Health, AP-HM, Aix-Marseille University, Marseille, France. ${ }^{4}$ Department of Research and Innovation, Support Unit for clinical research and economic evaluation, Assistance Publique - Hôpitaux de Marseille, Marseille 13385, France. ${ }^{5}$ Hôpital Sainte Marguerite, Assistance Publique - Hôpitaux de Marseille, Marseille cedex 9, France. 'Service de biologie médicale, CHI Poissy-Saint Germain, Poissy Cedex, France. 'EA 2493, UFR des sciences de la santé, 78180 Montigny-Le-Bretonneux, France.

Received: 11 May 2015 Accepted: 6 October 2015

Published online: 19 October 2015

\section{References}

1. Liu L, Johnson HL, Cousens S, Perin J, Scott S, Lawn JE, et al. Global, regional, and national causes of child mortality: an updated systematic analysis for 2010 with time trends since 2000. Lancet. 2012;379:2151-61.

2. Blondel B, Lelong N, Kermarrec M, Goffinet F. National Coordination Group of the National Perinatal S. Trends in perinatal health in France from 1995 to 2010. Results from the French National Perinatal Surveys. J Gynecol Obstet Biol Reprod (Paris). 2012;41:e1-e15.

3. Blencowe $H$, Cousens $S$, Oestergaard MZ, Chou D, Moller AB, Narwal R, et al. National, regional, and worldwide estimates of preterm birth rates in the year 2010 with time trends since 1990 for selected countries: a systematic analysis and implications. Lancet. 2012;379:2162-72.

4. Goldenberg RL, Culhane JF, lams JD, Romero R. Epidemiology and causes of preterm birth. Lancet. 2008;371:75-84.

5. Leitich $\mathrm{H}$, Kiss $\mathrm{H}$. Asymptomatic bacterial vaginosis and intermediate flora as risk factors for adverse pregnancy outcome. Best Pract Res Clin Obstet Gynaecol. 2007;21:375-90.

6. Menard JP, Mazouni C, Fenollar F, Raoult D, Boubli L, Bretelle F. Diagnostic accuracy of quantitative real-time PCR assay versus clinical and Gram stain identification of bacterial vaginosis. Eur J Clin Microbiol Infect Dis. 2010;29:1547-52.

7. Cartwright $C P$, Lembke BD, Ramachandran K, Body BA, Nye MB, Rivers CA, et al. Development and validation of a semiquantitative, multitarget PCR assay for diagnosis of bacterial vaginosis. J Clin Microbiol. 2012;50:2321-9.

8. Marconi C, Cruciani F, Vitali B, Donders GG. Correlation of Atopobium vaginae amount with bacterial vaginosis markers. J Low Genit Tract Dis. 2012;16:127-32.

9. Menard JP, Fenollar F, Henry M, Bretelle F, Raoult D. Molecular quantification of Gardnerella vaginalis and Atopobium vaginae loads to predict bacterial vaginosis. Clin Infect Dis. 2008:47:33-43.

10. Menard JP, Mazouni C, Salem-Cherif I, Fenollar F, Raoult D, Boubli L, et al. High vaginal concentrations of Atopobium vaginae and Gardnerella vaginalis in women undergoing preterm labor. Obstet Gynecol. 2010;115:134-40.

11. Bretelle F, Rozenberg P, Pascal A, Favre R, Bohec C, Loundou A, et al. High Atopobium vaginae and Gardnerella vaginalis Vaginal Loads are associated with preterm birth. Clin Infect Dis. 2015;60:860-7.

12. HAS. Prévention anténatale du risque infectieux bactérien néonatal précoce: recommandations, Septembre 2001. In: Servoces recommandations et références professionnelles. 2001. http://www.has-sante.fr/portail/upload/docs/ application/pdf/prevention_antenatale_du_risque_infectieux_bacterien__rec.pdf. Accessed 15 April 2015. 
13. Centers for Disease Control and Prevention (CDC). Sexually Transmitted Diseases Treatment Guidelines, 2010. In: Recommendations and Reports. 2010. http://www.cdc.gov/std/treatment/2010/std-treatment-2010rr5912.pdf. Accessed 15 April 2015.

14. Joesoef MR, Schmid G. Bacterial vaginosis. Clin Evid. 2005;13:1968-78.

15. Fredricks DN, Fiedler TL, Marrazzo JM. Molecular identification of bacteria associated with bacterial vaginosis. N Engl J Med. 2005;353:1899-911.

16. Shipitsyna E, Roos A, Datcu R, Hallen A, Fredlund H, Jensen JS, et al. Composition of the vaginal microbiota in women of reproductive agesensitive and specific molecular diagnosis of bacterial vaginosis is possible? PLoS One. 2013;8:e60670.

17. Menard JP, Fenollar F, Raoult D, Boubli L, Bretelle F. Self-collected vaginal swabs for the quantitative real-time polymerase chain reaction assay of Atopobium vaginae and Gardnerella vaginalis and the diagnosis of bacterial vaginosis. Eur J Clin Microbiol Infect Dis. 2012;31:513-8.

18. Kiss H, Pichler E, Petricevic L, Husslein P. Cost effectiveness of a screen-andtreat program for asymptomatic vaginal infections in pregnancy: towards a significant reduction in the costs of prematurity. Eur J Obstet Gynecol Reprod Biol. 2006;127:198-203.

19. AAA, ACOG. Guidelines for perinatal care. American Academy of Pediatrics. American College of Obstetricians and Gynecologists. 7th ed. Washington, DC: Elk Grove Village (IL); 2012.

20. Beaino G, Khoshnood B, Kaminski M, Marret S, Pierrat V, Vieux R, et al. Predictors of the risk of cognitive deficiency in very preterm infants: the EPIPAGE prospective cohort. Acta Paediatr. 2011;100:370-8.

21. Berbis J, Einaudi MA, Simeoni MC, Brevaut-Malaty V, Auquier P, d'Ercole C, et al. Quality of life of early school-age French children born preterm: a cohort study. Eur J Obstet Gynecol Reprod Biol. 2012;162:38-44.

22. Briggs AH, Gray AM. Power and sample size calculations for stochastic costeffectiveness analysis. Med Decis Making. 1998;18(2 Suppl):S81-92.

23. Kiss $H$, Petricevic L, Husslein P. Prospective randomised controlled trial of an infection screening programme to reduce the rate of preterm delivery. BMJ. 2004;329:371.

24. Goffinet F, Maillard F, Mihoubi N, Kayem G, Papiernik E, Cabrol D, et al. Bacterial vaginosis: prevalence and predictive value for premature delivery and neonatal infection in women with preterm labour and intact membranes. Eur J Obstet Gynecol Reprod Biol. 2003;108:146-51.

25. Petrou S, Khan K. Economic costs associated with moderate and late preterm birth: primary and secondary evidence. Semin Fetal Neonatal Med. 2012;17:170-8.

26. Schulz KF, Altman DG, Moher D, Group C. CONSORT 2010 Statement: updated guidelines for reporting parallel group randomised trials. Trials. 2010;11:32.

27. Drummond MF, Sculpher MJ, Torrance GW, O'Brien BJ, Stoddart GL. Methods for the economic evaluation of health care programmes. 3rd ed. Oxford: Oxford University Press; 2005.

28. Clements KM, Barfield WD, Ayadi MF, Wilber N. Preterm birth-associated cost of early intervention services: an analysis by gestational age. Pediatrics. 2007;119:e866-874.

29. Russell RB, Green NS, Steiner CA, Meikle S, Howse JL, Poschman K, et al. Cost of hospitalization for preterm and low birth weight infants in the United States. Pediatrics. 2007;120:e1-9.

30. Petrou $\mathrm{S}$. The economic consequences of preterm birth during the first 10 years of life. BJOG. 2005;112 Suppl 1:10-5.

31. Briggs $\mathrm{AH}$. Statistical approaches to handling uncertainty in health economic evaluation. Eur J Gastroenterol Hepatol. 2004;16:551-61.

32. Fenwick E, Claxton K, Sculpher M. Representing uncertainty: the role of costeffectiveness acceptability curves. Health Econ. 2001;10:779-87.

33. Brocklehurst P, Gordon A, Heatley E, Milan SJ. Antibiotics for treating bacterial vaginosis in pregnancy. Cochrane Database Syst Rev. 2013;1:CD000262.

34. Okun N, Gronau KA, Hannah ME. Antibiotics for bacterial vaginosis or Trichomonas vaginalis in pregnancy: a systematic review. Obstet Gynecol. 2005; 105:857-68

35. Nygren P, Fu R, Freeman M, Bougatsos C, Klebanoff M, Guise JM, et al. Evidence on the benefits and harms of screening and treating pregnant women who are asymptomatic for bacterial vaginosis: an update review for the U.S. Preventive Services Task Force. Ann Intern Med. 2008;148:220-33.

36. Lamont RF, Taylor-Robinson D. Review of the accuracy of various diagnostic tests for bacterial vaginosis to predict preterm birth (Honest et al., BJOG, May 2004). BJOG. 2005;112:259-60. author reply 260-251.
37. McGregor JA, French Jl, Parker R, Draper D, Patterson E, Jones W, et al. Prevention of premature birth by screening and treatment for common genital tract infections: results of a prospective controlled evaluation. Am J Obstet Gynecol. 1995;173:157-67.

38. Menard JP, Bretelle F. How can the treatment of bacterial vaginosis be improved to reduce the risk of preterm delivery? Womens Health (Lond Engl). 2012:8:491-3.

39. Bradshaw CS, Morton AN, Hocking J, Garland SM, Morris MB, Moss LM, et al. High recurrence rates of bacterial vaginosis over the course of 12 months after oral metronidazole therapy and factors associated with recurrence. J Infect Dis. 2006;193:1478-86.

40. De Backer E, Verhelst R, Verstraelen H, Claeys G, Verschraegen G, Temmerman M, et al. Antibiotic susceptibility of Atopobium vaginae. BMC Infect Dis. 2006;6:51.

41. Svare JA, Schmidt H, Hansen BB, Lose G. Bacterial vaginosis in a cohort of Danish pregnant women: prevalence and relationship with preterm delivery, low birthweight and perinatal infections. BJOG. 2006;113:1419-25.

42. Behrman RE, Butler AS. Preterm birth: causes, consequences, and prevention. Washington (DC): National Academies Press (US); 2007.

43. Mangham LJ, Petrou S, Doyle LW, Draper ES, Marlow N. The cost of preterm birth throughout childhood in England and Wales. Pediatrics. 2009;123:e312-327.

44. Korvenranta E, Lehtonen L, Rautava L, Hakkinen U, Andersson S, Gissler M, et al. Impact of very preterm birth on health care costs at five years of age. Pediatrics. 2010;125:e1109-1114.

\section{Submit your next manuscript to BioMed Central and take full advantage of:}

- Convenient online submission

- Thorough peer review

- No space constraints or color figure charges

- Immediate publication on acceptance

- Inclusion in PubMed, CAS, Scopus and Google Scholar

- Research which is freely available for redistribution

Submit your manuscript at www.biomedcentral.com/submit 